\title{
Entornos personales de aprendizaje móvil: una revisión sistemática de la literatura
}

\section{Mobile Personal Learning Environments: A systematic literature review}

\author{
Patricio Humanante-Ramos \\ Universidad Nacional de Chimborazo, UNACH (Ecuador) \\ Francisco José García-Peñalvo \\ Universidad de Salamanca, USAL (España) \\ Miguel Conde-González \\ Universidad de León, ULE (España)
}

\section{Resumen}

Actualmente, los entornos personales de aprendizaje aparecen como espacios educativos centrados en el estudiante para superar los limitantes identificados en las plataformas de aprendizaje institucional. Sin embargo, la mayoría de estudios publicados se basan únicamente en el uso de computadores sin considerar los dispositivos electrónicos móviles. El presente trabajo analiza la producción científica y académica sobre los Entornos Personales de Aprendizaje móvil en la Educación Superior. Desde el punto de vista metodológico, el estudio se realizó por medio de una revisión sistemática de la literatura utilizando varios recursos como: Scopus, Web Of Science, Google Scholar, entre otros. Se recogieron inicialmente una gran cantidad de documentos entre los años 2000 y 2016, a los cuales se aplicaron varios criterios de selección y de calidad, obteniéndose un número reducido de estudios que se analizaron de manera cualitativa. Los resultados fueron descritos en categorías de acuerdo a las preguntas de investigación planteadas, donde se incluyeron aquellos estudios sobre dispositivos móviles y entornos personales de aprendizaje, los que muestran proyectos de plataformas integradas, las investigaciones donde se usan herramientas externas con un enfoque más pedagógico, algunos aportes teóricos y los que evalúan estas innovaciones. En conclusión, los entornos personales de aprendizaje móvil se muestran como escenarios tecnológicos de reciente introducción en la educación universitaria y, aunque se han encontrado resultados satisfactorios, se requiere más investigación para poder establecer generalizaciones replicables en otros contextos.

Palabras clave: aprendizaje asistido por ordenador; autoaprendizaje; tecnologías de la información y de la comunicación; aprendizaje móvil; entornos personales de aprendizaje. 


\begin{abstract}
Nowadays personal learning environments appear as student-centered educational spaces to overcome the identified constraints in the institutional learning platforms. However, most of the published research is based only on the use of computers, without considering mobile electronic devices. This article analyses the scientific and academic production on mobile personal learning environments in Higher Education. From a methodological point of view, the research presented here was conducted through a systematic literature review using various resources such as Scopus, Web of Science, Google Scholar, among others. A large number of documents from 2000 to 2016 were initially collected. Subsequently, selection and quality criteria were applied, and a few studies were obtained that were qualitatively analyzed. The results were described in categories according to the original research questions, including research works on mobile devices and personal learning environments, publications on projects of integrated platforms, research where external tools are used with a pedagogical approach, theoretical contributions, and research that evaluates these innovations. In conclusion, mobile personal learning environments are shown as technological scenarios that have been recently introduced in Higher Education. Although successful results have been found, more research is needed to establish generalizations that might be replicable in other contexts.
\end{abstract}

Keywords: computer aided learning; self-learning; information and communication technologies; mobile learning; personal learning environments.

Las plataformas institucionales gestionadas a través de los sistemas de gestión de aprendizaje (Learning Management Systems o LMS) están presentes en casi la totalidad de instituciones de educación superior en todo el mundo (Babo y Azevedo, 2009; García-Peñalvo y Seoane Pardo, 2015), como es el caso de Ecuador, país latinoamericano donde las Universidades y Escuelas Politécnicas han propiciado el uso de los LMS como complemento a las clases presenciales y para la gestión de sus cursos virtuales o en línea. Sin embargo, la mayoría de cursos diseñados en estas plataformas se realizan de acuerdo a un plan de estudios predeterminado, el cual es el mismo para todos los estudiantes sin tomar en consideración las diferentes aptitudes, estilos y necesidades de aprendizaje, por lo que los estudiantes realizan una buena parte de sus actividades académicas, fuera de estos entornos institucionales (Downes, 2010) a través de otros recursos como los de la Web 2.0 y los dispositivos electrónicos móviles (Attwell, 2007; Meléndez Tamayo, 2013; Humanante-Ramos, García-Peñalvo y Conde-González, 2015).

En este sentido, para superar las deficiencias encontradas con la adopción de los LMS, aparecen los Entornos Personales de Aprendizaje o PLE (Personal Learning Environments) como espacios educativos centrados en el estudiante (Adell y Castañeda, 2010, 2013).

Así, la comunidad científica y académica, por un lado, reporta varias experiencias de implementaciones PLE en universidades y, por otro lado, publica estudios sobre 
la integración de herramientas Web 2.0 y dispositivos móviles, pero no se recogen estudios donde combinen estas prácticas educativas.

Por ejemplo, Buchem, Attwell y Torres (2011) reúnen algunos trabajos sobre PLE, centrándose en sus rasgos característicos a partir de la Teoría de la Actividad, describiendo sus elementos claves y las relaciones entre ellos.

Sin embargo, en la mayoría de estos trabajos se asumen como medios tecnológicos únicamente a los ordenadores, sin considerar los dispositivos electrónicos móviles ampliamente utilizados (International Telecommunications Union, 2016), donde los teléfonos inteligentes y tabletas constituyen los dispositivos preferidos por los universitarios (Bomhold, 2013).

Otro aporte corresponde al trabajo de Conde-González (2012), donde se presenta el estado del arte acerca de la interoperabilidad entre LMS y PLE, en el cual una de las preguntas de investigación indaga sobre lo que está sucediendo con los PLE más allá de los ordenadores, es decir, en otros contextos como los móviles, sin embargo, el autor informa que son pocas las investigaciones de este tipo.

En este sentido, el presente trabajo investiga y analiza la producción científica y académica sobre los Entornos Personales de Aprendizaje Móvil o mPLE (mobile Personal Learning Environments) (Attwell, Cook y Ravenscroft, 2009; CondeGonzález, García-Peñalvo, Alier y Piguillem, 2012; Ignatko y Zielasko, 2012) en la Educación Superior, cuyos resultados permitirán diseñar e implementar un mPLE en un contexto real de aprendizaje en una universidad Latinoamericana.

\section{METODOLOGÍA}

Este trabajo se apoya en la metodología propuesta por Kitchenham (2004) para realizar revisiones sistemáticas de literatura o SLR (Systematic Literature Review), así como en los aportes de Okoli y Schabram (2010), a partir de los cuales se siguió una serie de pasos, como se ilustra en la figura 1.

El desarrollo de cada uno de los pasos mencionados anteriormente permitió realizar la SLR de una manera sistémica. A continuación, se detalla el protocolo seguido, el cual puede ser reproducible en otros contextos. 
Figura 1. Pasos para realizar una SLR (Okoli y Schabram, 2010)

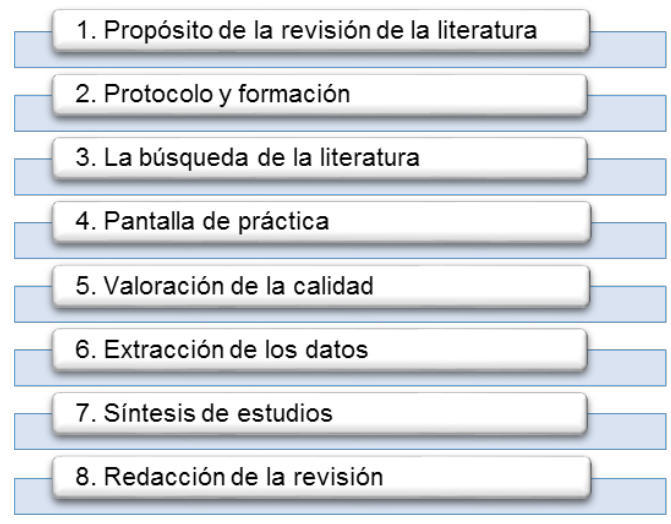

\section{Protocolo de revisión}

El protocolo, a través de la cual se condujo el proceso de revisión, parte con una definición de las preguntas de investigación, las estrategias de búsqueda y los criterios de inclusión y de evaluación de la calidad. A continuación, se describen cada uno de ellos:

\section{Preguntas de investigación}

Para esta revisión de la literatura, se plantearon las siguientes preguntas de investigación:

a. ¿Cuáles son las principales investigaciones sobre PLE en instituciones de Educación Superior, donde exista la inclusión de dispositivos móviles?

b. ¿Qué enfoque, técnico o pedagógico, tienen los PLE o mPLE en la Educación Superior?

c. ¿Cuáles son las propuestas de arquitectura y/o diseño para la implementación de los PLE o mPLE en contextos universitarios?

d. ¿Qué y cómo se evalúa la introducción de los PLE o mPLE en contextos universitarios? 


\section{Estrategias de búsqueda}

El proceso de búsqueda de información se realizó tanto de forma manual como electrónica. Así para el primer caso se recurrió a las bibliotecas de la Universidad de Salamanca y de la Universidad Pontificia de Salamanca (España). Para la búsqueda de la información de manera electrónica se accedió a una serie de sitios y recursos, los cuales se detallan en la tabla 1.

En vista de que la mayoría de sitios mencionados en la Tabla 1 publican sus contenidos en idioma inglés, se utilizaron los términos de búsqueda tanto en español como en inglés con sus respectivos acrónimos y combinaciones, entre los cuales se puede mencionar: entornos personales de aprendizaje, personal learning environments, PLE, entornos personales de aprendizaje móvil, mobile personal learning environment, mPLE combinados con los términos educación superior, educación universitaria, higher education, university education, respectivamente.

Estos términos de búsqueda fueron utilizados tanto en las secciones de títulos como en los resúmenes y en las palabras clave (keywords), y fueron complementados mediante intersecciones y uniones a través de los operadores AND y OR con el fin de seleccionar conjuntos de datos específicos.

\section{Criterios de inclusión y de evaluación de la calidad}

Las diferentes búsquedas dieron como resultado un número considerable de recursos (1554 documentos, entre los años 2000 y 2016), de los cuales se eliminaron aquellos que no incluyeron todos los términos de búsqueda, los de términos homónimos de otras áreas de estudio y los repetidos. De esta forma se filtraron 640 recursos, sin embargo, no todos los documentos fueron un aporte directo para responder a las preguntas planteadas en esta revisión.

Tabla 1. Fuentes consultadas para la realización de la SLR

\begin{tabular}{|c|c|}
\hline Fuente & Dirección Web \\
\hline BD SCOPUS & https://www.scopus.com/ \\
\hline Web Of Science & https://www.accesowok.fecyt.es/ \\
\hline $\begin{array}{l}\text { Herramientas especializadas de } \\
\text { Google }\end{array}$ & $\begin{array}{l}\text { http://scholar.google.es/ (Google Académico) } \\
\text { http://books.google.es/ (Google Libros) }\end{array}$ \\
\hline $\begin{array}{l}\text { Repositorios documentales, de } \\
\text { tesis y de artículos de revistas }\end{array}$ & $\begin{array}{l}\text { http://www.tesisenred.net/ } \\
\text { http://digital.csic.es/ } \\
\text { http://oatd.org/ } \\
\text { http://gredos.usal.es/jspui// }\end{array}$ \\
\hline
\end{tabular}




\begin{tabular}{|c|c|}
\hline Fuente & Dirección Web \\
\hline Actas específicas de congresos & $\begin{array}{l}\text { Technological Ecosystems for Enhancing } \\
\text { Multiculturality (http://teemconference.eu/) } \\
\text { Interaction Design in Educational Environments (http:// } \\
\text { idee-workshop.org/) } \\
\text { PLE Conference, en sus ediciones } 2010 \text { al } 2015 \\
\text { IEEE International Conference on Advanced Learning } \\
\text { Technologies (ICALT), en sus ediciones 2011-2012-2013 }\end{array}$ \\
\hline Varias revistas especializadas & 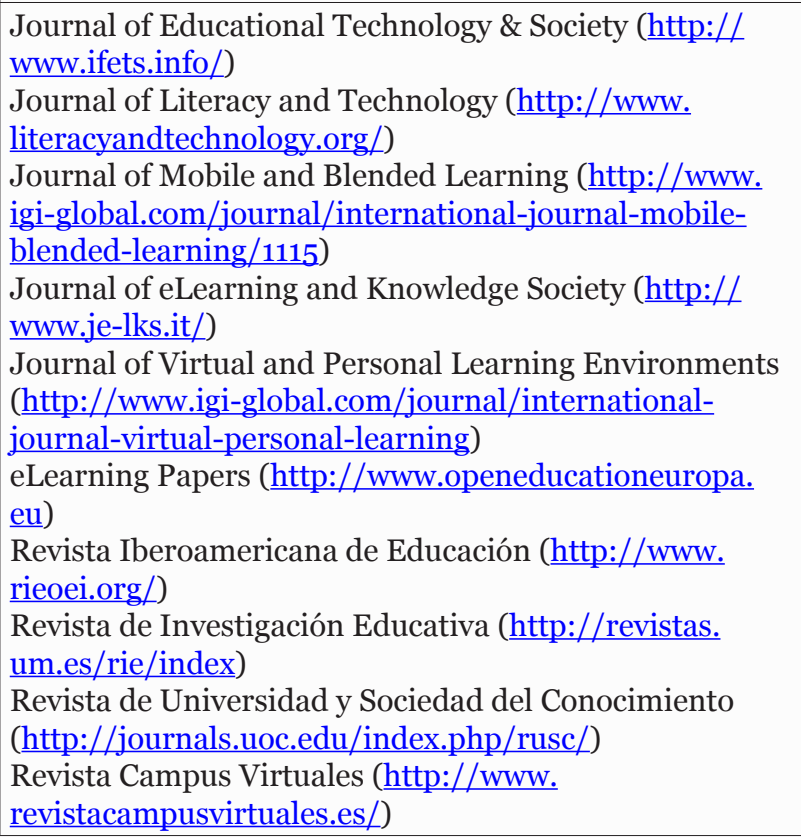 \\
\hline
\end{tabular}

\section{Elaboración propia}

En esta parte, fue necesario aplicar algunos criterios de inclusión tomando como referencia lo publicado por Buela-Casal (2003) y Conde-González (2012). Así, en esta parte se seleccionaron solo aquellas publicaciones que cumplieron los siguientes criterios:

- Que incluyan uno o más términos de búsqueda de acuerdo a los temas expuestos en las preguntas de investigación, con una estructura adecuada y que aporten alguna iniciativa de implementación PLE con tecnologías móviles.

- Que presenten conclusiones coherentes desde el punto de vista tecnológico, pedagógico o metodológico.

- Que sean revisiones críticas debidamente justificadas, de uno o más términos de búsqueda de acuerdo a los temas expuestos en las preguntas de investigación. 
De esta forma, se filtraron 208 documentos, los cuales fueron posteriormente evaluados por tres investigadores utilizando una escala de 1 a 5 según los criterios de calidad mostrados en la tabla 2.

Tabla 2. Criterios para evaluar la calidad de las publicaciones

\begin{tabular}{|c|c|}
\hline Puntuación & Criterio \\
\hline 1 & Constituyen estudios bibliográficos descriptivos sin mayor profundidad. \\
\hline 2 & Son aportes teóricos que proponen nuevos enfoques o tendencias. \\
\hline 3 & $\begin{array}{l}\text { Incluyen propuestas de diseño y/o implementación debidamente } \\
\text { fundamentadas. }\end{array}$ \\
\hline 4 & $\begin{array}{l}\text { Presentan experiencias prácticas de implementación sobre contextos reales } \\
\text { de aprendizaje. }\end{array}$ \\
\hline 5 & $\begin{array}{l}\text { A más de lo contemplado en el nivel } 4 \text { evalúan dichas implementaciones, } \\
\text { presentando resultados claros y replicables. }\end{array}$ \\
\hline
\end{tabular}

Elaboración propia

Una vez puntuados los documentos por cada revisor, se procedió a calcular los promedios para, finalmente, seleccionar solamente aquellos que alcanzaron valores mayores o iguales a 3. Así, el nuevo registro estuvo conformado por 117 estudios a partir de los cuales se realizó el proceso de extracción de datos.

\section{Procedimiento de extracción y síntesis de la información}

Luego de haber seleccionado únicamente los recursos considerados como idóneos para la elaboración de la revisión sistemática final, se procedió con la extracción de la información. Para esto se elaboró una matriz de extracción, diseñada en función de las preguntas de investigación y que incluyó para cada documento los siguientes datos: un código (número secuencial usado por el investigador para referenciar el recurso en la matriz de revisión), el título, los autores, el resumen, el año de publicación, el tipo de documento (artículo, comunicación, capítulo de libro, etc.), las palabras clave, los objetivos, la metodología, el diseño o propuesta (en caso de incluir, así como las tecnologías o herramientas usadas para su implementación), los resultados, las conclusiones, las observaciones y el análisis personal del investigador después de la lectura del documento.

A partir de esta información se procedió a realizar la síntesis, combinando la información extraída de cada documento con el fin de contar con una visión integral de los estudios, y analizar cómo estos dan su aporte para encontrar respuestas a las preguntas de investigación planteadas; comparándolos, contrastándolos y analizándolos de forma cualitativa (Okoli y Schabram, 2010). 


\section{RESULTADOS}

La distribución por años de los documentos inicialmente filtrados (640) se presenta en la figura 2, observándose un crecimiento ascendente a partir del 2008, siendo el año 2016 donde se ha publicado más, aproximadamente 14 veces más que en el 2008.

Figura 2. Publicaciones por años

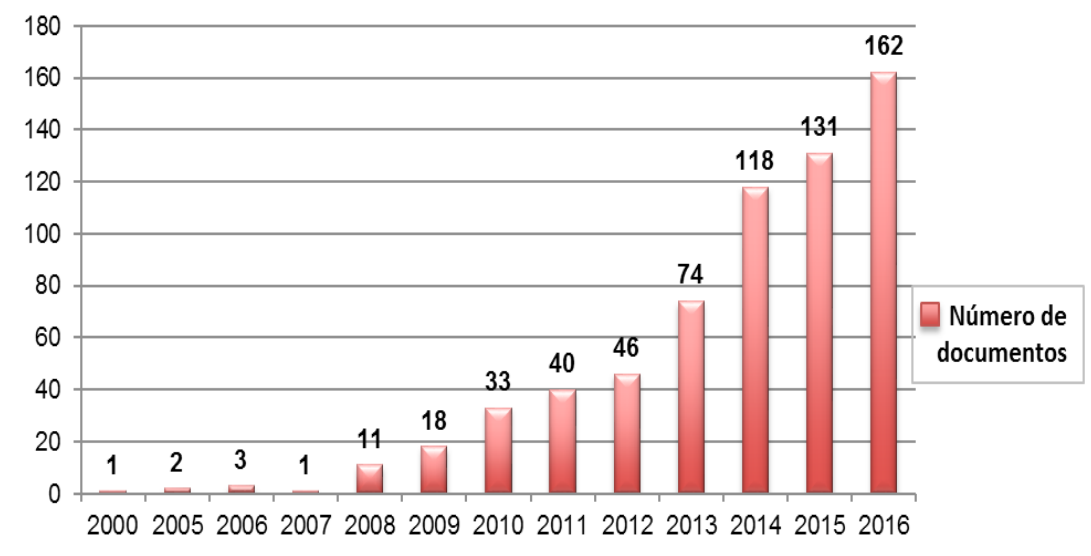

Una distribución por categorías de acuerdo a las preguntas de investigación muestra que la mayor parte de estudios son aquellos donde se usan herramientas externas para implementar los PLE con una orientación más pedagógica y, en menor número, aparecen los trabajos que relacionan PLE con dispositivos móviles (figura $3)$.

Figura 3. Publicaciones por categorías específicas

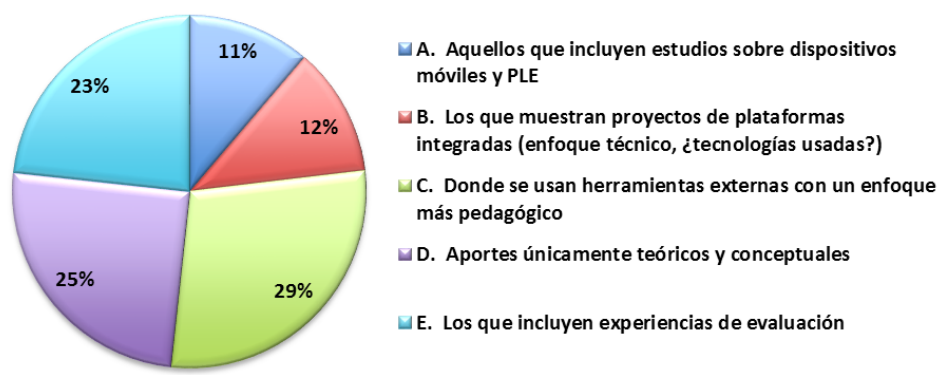


A continuación, se describen de forma cualitativa los estudios seleccionados de acuerdo a las preguntas de investigación inicialmente planteadas.

\section{a. ¿Cuáles son las principales investigaciones sobre PLE en instituciones de Educación Superior, donde exista la inclusión de dispositivos móviles?}

Respecto a la primera pregunta, se observa que los móviles vistos como PLEs se presentan como herramientas de apoyo pedagógico tanto a nivel individual como grupal. Se incluyen estudios donde se aprovechan las características multimediales de los móviles para el aprendizaje de idiomas. En otro grupo de trabajos, se busca la integración de los móviles con las plataformas institucionales para encontrar un punto de intersección entre lo formal y lo informal (García-Peñalvo, CondeGonzález, Alier y Casany, 2011), a través del uso de widgets y herramientas de software libre. Otros proyectos son una evolución de proyectos PLE basados en el uso de ordenadores y que, para asegurar su accesibilidad desde contextos móviles, han tenido que ampliarse hacia nuevas versiones (Taraghi, 2012; García-Peñalvo, Conde-González y Moreno García, 2013).

Por último, se encuentran trabajos en fases iniciales de investigación, algunos en universidades latinoamericanas, lo cual indica que en estos contextos educativos el tema de PLE y dispositivos móviles es de reciente introducción. Las investigaciones de este grupo se presentan en la tabla 3.

Tabla 3. Investigaciones sobre PLE en instituciones de educación superior donde se incluyan estudios sobre dispositivos móviles

\section{Descripción}

Como herramientas de uso pedagógico tanto a nivel individual como grupal.

Específicas para determinadas áreas del conocimiento, presentadas como mPLE.

\section{Documentos}

(Chan, Corlett, Sharples, Ting y Westmancott, 2005), (Andone, Vasiu, Ternauciuc y Dragulescu, 2010), (Punjabi, Tung y Lin, 2013), (Giemza, Malzahn y Hoppe, 2013). (Ogata et al., 2010), (Perifanou, 2011), (Thüs, Akbari y Schroeder, 2011), (Uosaki et al., 2011), (Attwell, Heinemann, Kamarainen, y Deitmer, 2013), (Li, Ogata, Hou, Uosaki y Mouri, 2013), (Aladjem y Nachmias, 2014), (Fok et al., 2014), (Greven, Chatti, Thüs y Schroeder, 2014), (Chatti, Thüs, Greven y Schroeder, 2015, 2016), (Gheorghiu y Ştefan, 2015), (Lopez, Zorrozua, Ruiz, Rodriguez-Artacho y Gil, 2016), (Humanante-Ramos, 2016). 


\begin{tabular}{|l|l|}
\hline \multicolumn{1}{|c|}{ Descripción } & \multicolumn{1}{c|}{ Documentos } \\
$\begin{array}{l}\text { Que buscan la integración } \\
\text { de los entornos } \\
\text { institucionales dentro del } \\
\begin{array}{l}\text { PLE y/o aquellas basadas } \\
\text { en el uso de widgets. }\end{array}\end{array}$ & $\begin{array}{l}\text { Garany-Guerrero y Piguillem-Pochalva 2010), (Conde-González, } \\
\text { Koulocheri y Xenos, 2011), (Casany et al., 2012), (Conde- } \\
\text { González, García-Peñalvo, Alier y Piguillem, 2012), (Conde- } \\
\text { González, García-Peñalvo, Alier y Piguillem, 2013), (Conde- } \\
\text { González, García-Peñalvo, Alier-Forment, Casany-Guerrero } \\
\text { y Piguillem-Poch, 2013), (García-Peñalvo y Conde-González, } \\
\text { 2015). }\end{array}$ \\
\hline $\begin{array}{l}\text { Propuestas en } \\
\text { fases iniciales de } \\
\text { implementación. }\end{array}$ & $\begin{array}{l}\text { (Barrios, Fernández, Godoy y Mariño, 2012), (Cataldi, } \\
\text { Méndez, Dominighini y Lage, 2012), (Van y Moes, 2016). }\end{array}$ \\
\hline
\end{tabular}

Elaboración propia

\section{b. ¿Qué enfoque, técnico o pedagógico, tienen los PLE o mPLE en la Educación Superior?}

Se puede observar que existen investigaciones que estudian la incorporación de los PLE o mPLE en la Educación Superior tanto desde el punto de vista técnico, como también desde el enfoque pedagógico.

Así, para el primer caso se encuentran los desarrollos de plataformas de software o sistemas informáticos que integran PLE con LMS. Estos trabajos resultan interesantes, ya que los LMS por si solos resultan limitados para soportar los nuevos enfoques educativos centrados en el estudiante pero que, al estar presentes en la mayoría de universidades, deberían ser integrados.

Desde el punto de vista técnico, las implementaciones a través de widgets es la forma común para estructurar los PLEs, siendo la capacidad de personalización y la facilidad de uso de estos nuevos entornos lo que marca sus diferencias. Se describen también frameworks de servicios para permitir la comunicación entre el contexto institucional (LMS, CMS) y el contexto personal (herramientas web 2.0, dispositivos móviles, etc.), adoptando arquitecturas orientadas a servicios (Services Oriented Architecture - SOA) (Casany, Alier, Conde-González y García-Peñalvo, 2009), así como tecnologías centradas en la nube (cloudcomputing) (Beloudane y Belalem, 2015).

En otros trabajos, se destaca la construcción de los PLEs a partir de las infraestructuras institucionales, como los PLEs potenciados institucionalmente (iPLE), el habilitador PLE institucional (iPLEe), el PLE híbrido institucional (HIPLE), por mencionar algunos. Las investigaciones de este grupo se presentan en la tabla 4 .

Sin embargo, los PLEs también pueden ser diseñados desde un enfoque pedagógico. En este tipo de innovaciones educativas, para la implementación del 
PLE no se requiere el desarrollo de una nueva plataforma de software, sino que se aprovechan los recursos disponibles en la web.

Tabla 4. Estudios sobre PLEs en contextos universitarios con enfoque técnico

\begin{tabular}{|c|c|}
\hline Descripción & Documentos \\
\hline $\begin{array}{l}\text { Implementan MashUp } \\
\text { basado en widgets y } \\
\text { otras relacionadas con el } \\
\text { proyecto europeo ROLE } \\
\text { hacia una facilitación del } \\
\text { aprendizaje auto-regulado. }\end{array}$ & $\begin{array}{l}\text { (Mödritscher y Wild, 2009), (Taraghi, Ebner y Schaffert, } \\
\text { 2009), (Mödritscher, 2010), (Friedrich et al., 2011), (Ebner, } \\
\text { Schön, Taraghi, Drachsler y Tsang, 2011), (Kravcik y Klamma, } \\
\text { 2012), (Nussbaumer, Kravcik y Albert, 2012), (Auinger, } \\
\text { Nedbal, Holzinger, Scerbakov y Ebner, 2013), (Hernandez- } \\
\text { Rizzardini, Linares, Mikroyannidis y Schmitz, 2013), (Leone, } \\
\text { 2013), (Nussbaumer et al., 2014). }\end{array}$ \\
\hline $\begin{array}{l}\text { Frameworks basados en } \\
\text { arquitecturas orientadas } \\
\text { a servicios o frameworks } \\
\text { para desarrollar nuevas } \\
\text { funcionalidades y/o para } \\
\text { acceder a los LMS desde } \\
\text { dispositivos móviles. }\end{array}$ & $\begin{array}{l}\text { (Wild, Mödritscher y Sigurdarson, 2008), (Casany, Alier, } \\
\text { Conde-González y García-Peñalvo, 2009), (García-Peñalvo, } \\
\text { Conde-González, Alier y Casany, 2011), (Conde-González, } \\
\text { 2012), (Conde-González, García-Peñalvo, Alier y Mayol, 2012), } \\
\text { (Conde-González, García-Peñalvo, Rodríguez Conde y Alier- } \\
\text { Forment, 2012), (Simões, Rodrigues y Costa, 2013), (Simões, } \\
\text { Rodrigues y de la Torre, 2013), (Conde-González et al., 2014), } \\
\text { (Conde-González, García-Peñalvo, Rodríguez Conde, Alier- } \\
\text { Forment y García-Holgado, 2014), (Hung, Lam, Wong y Chan, } \\
\text { 2015), (Joo, Kim y Kim, 2016). }\end{array}$ \\
\hline $\begin{array}{l}\text { Integrando la tecnología } \\
\text { Cloud Computing. }\end{array}$ & $\begin{array}{l}\text { (Simões, Rodrigues, Costa y Proenca, 2012), (Mikroyannidis } \\
\text { et al., 2013), (Chen, Al-Bayatti y Siewe, 2016), (Wu y Chang, } \\
\text { 2016). }\end{array}$ \\
\hline $\begin{array}{l}\text { Iniciativas de PLEs } \\
\text { institucionales. }\end{array}$ & $\begin{array}{l}\text { (Casquero, Portillo, Ovelar, Benito y Romo, 2010), (Casquero, } \\
\text { Portillo, Ovelar, Romo y Benito, 2010), (Moccozet, Benkacem, } \\
\text { Burgi, Platteaux y Gillet, 2012), (Casquero, 2013), (Morán- } \\
\text { López, 2013), (Coll, Engel, Saz y Bustos, 2014), (Hermans, } \\
\text { Kalz y Koper, 2014), (Moccozet, Benkacem, Platteaux y } \\
\text { Foerster, 2014), (Moccozet y Tardy, 2014), (Saz, Engel y Coll, } \\
\text { 2016), (Casquero, Ovelar, Romo, Benito y Alberdi, 2016). }\end{array}$ \\
\hline $\begin{array}{l}\text { Uso de portafolios } \\
\text { digitales y su integración } \\
\text { con LMS. }\end{array}$ & $\begin{array}{l}\text { Oliveira y Moreira, 2010), (Salinas, Marín y Escandell, 2011), } \\
\text { (Terkowsky, May, Haertel y Pleul, 2012), (García-Planas y } \\
\text { Taberna-Torres, 2014), (Terkowsky, Haertel, Bielski y May, } \\
\text { 2014). }\end{array}$ \\
\hline
\end{tabular}

Elaboración propia

Así, en los trabajos estudiados, por un lado, se promueve el uso de herramientas web 2.0 para actividades educativas que propicien la reflexión en el aprendizaje, aunque se observa el escaso control de las instituciones en estas nuevas formas de aprender. 
En otros estudios, se busca mejorar los actuales MOOC (Massive Open Online Course) desde un enfoque conectivista (cMOOC) (Fidalgo-Blanco, Sein-Echaluce y García-Peñalvo, 2015; Adell y Castañeda, 2013), donde el proceso de adquisición del conocimiento si bien responde a un trabajo autónomo, motivado y autorregulado, pero que se nutre de la participación y colaboración en red. Las investigaciones de este grupo se presentan en la tabla 5 .

Tabla 5. Estudios sobre PLEs en contextos universitarios con enfoque pedagógico

\begin{tabular}{|l|l|}
\hline \multicolumn{1}{|c|}{ Descripción } & \multicolumn{1}{c|}{ Documentos } \\
\hline $\begin{array}{l}\text { Uso de herramientas } \\
\text { Web 2.o diversas (redes } \\
\text { sociales, blogs, microblogs, } \\
\text { wikis, marcadores sociales, } \\
\text { recomendadores y otras) }\end{array}$ & $\begin{array}{l}\text { (Castañeda y Sánchez, 2009), (Castañeda y Soto, 2010), } \\
\text { (Camacho y Guilana, 2011), (Arquero y Romero-Frias, 2012), } \\
\text { (Martini y Cinque, 2012), (Tomberg, Laanpere, Ley y Normak, } \\
\text { 2013), (Díaz-Redondo, Fernández-Vilas, Pazos-Arias y Gil- } \\
\text { Solla, 2014), (Haworth, 2016), (Gutiérrez, Barriga, Ramírez- } \\
\text { Corona, Lopez-Malo y Palou, 2016), (Prendes, Castañeda, } \\
\text { Gutiérrez y Román, 2016) }\end{array}$ \\
\hline $\begin{array}{l}\text { Uso de determinadas } \\
\text { herramientas hacia el } \\
\text { aprendizaje autorregulado, } \\
\text { personalizado y } \\
\text { colaborativo }\end{array}$ & $\begin{array}{l}\text { (Frydenberg, 2006), (Edirisingha, Salmon y Fothergill, 2007), } \\
\text { (Peacock, Fellows y Eustace, 2007), (Elgort, Smith y Toland, } \\
\text { 2008), (English y Duncan-Howell, 20o8), (McLoughlin y } \\
\text { Lee, 2010), (Marín-Juarros, Negre-Bennasar y Pérez-Garcias, } \\
\text { 2014), (Matzat y Vrieling, 2016) }\end{array}$ \\
\hline $\begin{array}{l}\text { Experiencias MOOC, } \\
\text { cMOOC apoyados en } \\
\text { herramientas Web 2.0 }\end{array}$ & (Kop y Foumier, 2013), (Saadatmand y Kumpulainen, 2013) \\
\hline
\end{tabular}

Elaboración propia

\section{c. ¿Cuáles son las propuestas de arquitectura y/o diseño para la implementación de los PLE o mPLE en contextos universitarios?}

Varias propuestas de diseño y/o arquitectura se basan en el uso de servicios gestionados a través de un framework o de un entorno de servicios. En estas propuestas, el usuario accedería a la información a través de herramientas específicas o interfaces que garanticen el acceso desde varios contextos. Sin embargo, las grandes limitantes para universalizar este tipo de soluciones están relacionados con la diversidad de tecnología (hardware, sistemas operativos, aplicaciones, etc.) asociadas a los dispositivos que usan los estudiantes, como también por la variedad de plataformas institucionales existentes, ya que, en estas propuestas, la integración con los VLEs (Virtual Learning Environments) resulta fundamental.

Otras propuestas de diseño se basan en el uso de herramientas, servicios y recursos digitales, cuya selección depende de las funcionalidades que deben aportar 
los PLE, es decir, la manera de acceder a contenidos, los procesos de reflexión y las formas de interacción con otros (Adell y Castañeda, 2010) deberían orientar la selección de tal o cual herramienta. Los aportes referenciados en este trabajo proporcionan importantes tendencias de uso de determinadas herramientas por los jóvenes universitarios.

Por otro lado, el aprendizaje auto-regulado, sensible al contexto y permanente, motiva el desarrollo de propuestas PLE, donde a corto plazo, se ve como una oportunidad el aprovechar los contextos derivados de las ciudades inteligentes (smartcities), especialmente, aquellos avances tecnológicos relacionados con la geolocalización (JooNagata, García-Bermejo Giner y Martínez Abad, 2016), la realidad aumentada (De la Torre Cantero, Martin-Dorta, Saorín Pérez, Carbonel Carrera y Contero González, 2013) y el etiquetado móvil, por mencionar algunos.

Las investigaciones de este grupo se presentan en la tabla 6.

Tabla 6. Propuestas de diseño y/o arquitectura para implementación de PLE o mPLE

\begin{tabular}{|l|l|}
\hline \multicolumn{1}{|c|}{ Descripción } & \multicolumn{1}{c|}{ Documentos } \\
\hline $\begin{array}{l}\text { Arquitecturas o modelos para } \\
\text { el diseño de PLE }\end{array}$ & $\begin{array}{l}\text { (Johnson, Hollins, Wilson y Liber, 2006), (Alharbi, Platt y } \\
\text { Al-Bayatti, 2012), (Dabbagh y Kitsantas, 2012), (Castañeda } \\
\text { y Adell, 2013), (Manso-Vazquez y Llamas-Nistal, 2013), } \\
\text { (Humanante-Ramos, García-Peñalvo y Conde-González, } \\
\text { 2016), (López, Builes y Puche, 2016) }\end{array}$ \\
\hline $\begin{array}{l}\text { Describen las funcionalidades } \\
\text { del PLE }\end{array}$ & $\begin{array}{l}\text { (Milligan et al., 2006), (Attwell, 2007), (Adell y Castañeda, } \\
\text { 2010) }\end{array}$ \\
\hline $\begin{array}{l}\text { Nuevos escenarios para el } \\
\text { desarrollo PLE }\end{array}$ & $\begin{array}{l}\text { (Buchem y Pérez-Sanagustín, 2013), (Cabero-Almenara y } \\
\text { Marín, 2013), (Marín, Lizana y Salinas, 2014) }\end{array}$ \\
\hline
\end{tabular}

Elaboración propia

\section{d. ¿Qué y cómo se evalúa la introducción de los PLE o mPLE en contextos universitarios?}

La mayoría de evaluaciones PLE y mLearning corresponden a diseños mixtos que integran el enfoque cuantitativo y cualitativo para el tratamiento de la información, siendo pocas las experiencias que evalúan estos escenarios educativos desde un único corte cuantitativo o cualitativo.

Por último, algunos trabajos analizados orientan sobre varios aspectos a tener en cuenta para medir la efectividad de las implementaciones PLE y mLearning en la educación universitaria. En este sentido, las principales variables que se estudian son: creatividad, nivel de innovación, reflexión, auto-regulación, autonomía, motivación, flexibilidad, movilidad, interacción, percepción de utilidad y facilidad de uso.

Un resumen de las principales investigaciones donde se incluyen experiencias de evaluación se detallan en la tabla 7. 
Tabla 7. Experiencias de evaluación de PLE o mPLE

\begin{tabular}{|c|c|}
\hline Descripción & Documentos \\
\hline $\begin{array}{l}\text { Evaluaciones de los PLE en } \\
\text { contextos móviles }\end{array}$ & $\begin{array}{l}\text { (Conde-González y García-Peñalvo, 2014), (García-Peñalvo } \\
\text { y Conde-González, 2014) }\end{array}$ \\
\hline $\begin{array}{l}\text { Evaluaciones de } \\
\text { implementaciones PLE y } \\
\text { mLearning }\end{array}$ & $\begin{array}{l}\text { (Santamaria, 2010), (Suki y Suki, 2011), (Cheon, Lee, Crooks } \\
\text { y Song, 2012), (Kroop, 2013), (Rahimi, Van den Berg y } \\
\text { Veen, 2013), (Almaiah y Jalil, 2014), (Alrasheedi y Capretz, } \\
\text { 2015),(Wilkinson y Barter, 2015), (Wong, Wang, Ng y Kwan, } \\
\text { 2015), (Briz Ponce, 2016) }\end{array}$ \\
\hline
\end{tabular}

Elaboración propia

Para facilitar el manejo de este documento, las referencias bibliográficas citadas en las tablas 3, 4, 5, 6 y 7 están accesibles online (https://goo.gl/r5aZg9).

\section{DISCUSIÓN Y CONCLUSIONES}

El presente trabajo expone el estado de la cuestión sobre los Entornos Personales de Aprendizaje Móvil (mPLE) en la educación universitaria, para lo cual se desarrolló una revisión sistemática de la literatura a partir de investigaciones encontradas en diversas bases de datos científicas y repositorios documentales.

Así, de acuerdo a las preguntas de investigación planteadas para esta revisión, lo primero que interesaba conocer eran las principales investigaciones sobre PLE en instituciones de educación superior donde existiera la inclusión de dispositivos móviles. En este sentido, se encontraron varias evidencias científicas importantes. Sin embargo, las formas cómo se conciben, se diseñan y se implementan los PLE difieren unas de otras. Esto concuerda con las opiniones expresadas por algunos autores acerca de la diversidad de criterios existentes en la conceptualización del PLE (Johnson et al., 2006; Milligan et al., 2006; Adell y Castañeda, 2010; Ebner, Schön, Taraghi, Drachsler y Tsang, 2011; Castañeda y Adell, 2013).

Un dato importante a mencionar en esta parte es que la mayoría de trabajos corresponden a investigaciones realizadas en universidades europeas (aproximadamente el 72\%) y el resto en universidades de América y Asia, lo que indica que el diseño e implementación de los mPLE en las universidades latinoamericanas son de reciente introducción, existiendo en estos contextos un gran potencial de implementación para este tipo de proyectos.

En cuanto al enfoque adoptado para implementar los PLE o mPLE en las universidades, se observó dos corrientes conceptuales en los trabajos revisados: una más técnica (Casquero, Portillo, Ovelar, Benito y Romo, 2010; CondeGonzález, 2012; Türker y Zingel, 2008; van Harmelen, 2006; Wilson et al., 2006) y otra pedagógica (Adell y Castañeda, 2010; Cabero-Almenara y Vázquez-Martínez, 2013). Dentro de la primera corriente, se describen las propuestas orientadas al 
desarrollo de nuevas plataformas institucionales, desde donde los estudiantes diseñan sus PLE y aquellos proyectos que mejoran las infraestructuras existentes en las universidades, dotándoles de capacidades de personalización con el objetivo de superar los limitantes de los actuales LMS (Johnson et al., 2006; Ebner, Schön, Taraghil, Drachsler y Tsang, 2011; Dabbagh y Kitsantas, 2012; Conde-González, 2012; Tomberg et al., 2013). La mayoría de estas implementaciones se basan en el uso de widgets y mashUps de widgets, en arquitecturas orientadas a servicios y en tecnologías centradas en la nube (cloud computing).

En este sentido, se ve que muchas investigaciones persiguen la integración y la convivencia de las plataformas institucionales con los nuevos contextos de aprendizaje centrados en el estudiante que proponen los PLE, dotándoles además de movilidad y flexibilidad, al buscar el acceso y gestión de estos escenarios educativos desde los dispositivos móviles.

Desde una corriente pedagógica, donde el diseño del PLE no implica un nuevo desarrollo de plataformas, se observa que son los mismos estudiantes quienes seleccionan los recursos y herramientas (redes sociales, blogs, microblogs, wikis, marcadores sociales, recomendadores y otras de la Web 2.0) de acuerdo a sus preferencias y estilos de aprendizaje; propiciando así el desarrollo de su autonomía, autorreflexión y autocontrol. En estos escenarios, también se evidencia un limitado control de los procesos de aprendizaje por parte de las instituciones de educación superior, quienes dejan que los estudiantes gestionen libremente su aprendizaje a través de los PLE.

En este punto, resulta importante pensar en nuevos entornos tecnológicos, donde las universidades vuelvan a tener el control de estos procesos, a través de la integración de las herramientas y de los servicios externos que los estudiantes utilizan para aprender. A estos nuevos entornos institucionales la comunidad académica ha empezado a denominarles ecosistemas tecnológicos de aprendizaje (García-Holgado y García-Peñalvo, 2016), donde los estudiantes tienen acceso a los contenidos desde múltiples dispositivos y plataformas (Llorens-Largo, Molina, Compañ y Satorre, 2014).

Otro interrogante abordado a través de la presente SLR tuvo que ver con las propuestas de arquitectura y/o diseño para la implementación de los PLE o mPLE en contextos universitarios, donde se incluyen diversos modelos de diseño (frameworks, entornos de servicios, modelos, etc.); algunos todavía no implementados en contextos reales de aprendizaje, o que a su vez no han sido replicados en colectivos universitarios representativos. Esto abre nuevas posibilidades de experimentación en contextos educativos universitarios latinoamericanos, a partir de los diseños o modelos recopilados en esta SLR (Castañeda y Adell, 2013; Manso-Vazquez y LlamasNistal, 2013; Buchem y Pérez-Sanagustín, 2013; Marín, Lizana y Salinas, 2014); replicando buenas prácticas que permitan además realizar estudios comparados inter-universidades. 
En cuanto al último interrogante sobre la evaluación de los proyectos PLE en universidades, la revisión sistemática mostró que la mayoría de trabajos académicos responden a diseños de investigación mixtos, lo que concuerda con el planteamiento de autores, como Hernández, Fernández y Baptista (2010), cuando afirman que, debido a la complejidad de los fenómenos y problemas que enfrentan las ciencias en la actualidad, como es el caso de la educación, no sería adecuado abordarla desde una sola posición, sea esta cuantitativa o cualitativa, sino desde un enfoque mixto de investigación.

Además, en los documentos analizados para este último interrogante, las variables estudiadas son diversas y, en su mayoría, están relacionadas con las percepciones y actitudes de los estudiantes hacia estos escenarios educativos, siendo escasamente estudiados los niveles de aprendizaje y el rendimiento académico. Esto, de igual forma, abre nuevas posibilidades de investigación al momento de evaluar este tipo de implementaciones tecnológicas que apoyan la personalización del aprendizaje y la movilidad.

Finalmente, se puede decir que la presente SLR recoge aspectos técnicos, metodológicos y de evaluación que la comunidad científica y académica ha generado, en la última década, sobre experiencias de implementaciones PLE y mPLE a nivel universitario, y que sería pertinente considerarlos para futuras implementaciones, tomando en cuenta que estos escenarios tecnológicos son de reciente adopción y, aunque se han encontrado resultados satisfactorios en los estudios revisados, se requiere más investigación para poder establecer generalizaciones replicables en otras instituciones de educación superior.

\section{REFERENCIAS BIBLIOGRÁFICAS}

Adell, J., y Castañeda, L. (2010). Los Entornos Personales de Aprendizaje (PLEs): una nueva manera de entender el aprendizaje. En R. Roig Vila y M. Fiorucci (Ed.), Claves para la investigación en innovación y calidad educativas. La integración de las Tecnologías de la Información y la Comunicación y la Interculturalidad en las aulas (19-30). Alcoy: Marfil - Rome TRE Universita degli studi.

Adell, J., y Castañeda, L. (2013). El ecosistema pedagógico de los PLEs. En L. Castañeda y J. Adell (Ed.), Entornos Personales de Aprendizaje: Claves para el ecosistema educativo en red (29-51). Alcoy: Marfil.
Attwell, G. (2007). Personal Learning Environments - the future of eLearning? En eLearning Papers, 2(1), 1-8.

Attwell, G., Cook, J., y Ravenscroft, A. (2009). Appropriating Technologies for Contextual Knowledge: Mobile Personal Learning Environments. En M. D. Lytras, P. O. de Pablos, E. Damiani, D. Avison, A. Naeve y D. G. Horner (Ed.), Best Practices for the Knowledge Society. Knowledge, Learning, Development and Technology for All (15-25). Heidelberg: Springer.

Babo, R., y Azevedo, A. (2009). Learning management systems usage on higher education institutions. In Proceedings of the 13th International Business Information Management Association 
Conference, IBIMA 2009 (pp. 883-889). Marrakech, Marruecos.

Bomhold, C. R. (2013). Educational use of smart phone technology: A survey of mobile phone application use by undergraduate university students. Program, 47(4), 424-436. doi: http://doi. org/10.1108/PROG-01-2013-0003

Buchem, I., Attwell, G., y Torres, R. (2011). Understanding Personal Learning Environments: Literature review and synthesis through the Activity Theory lens. En Proceedings of the PLE Conference 2011 (pp. 1-33). Southampton, Reino Unido.

Buchem, I., y Pérez-Sanagustín, M. (2013). Personal Learning Environments in Smart Cities: Current Approaches and Future Scenarios. eLearning Papers, 35(1), 1-14.

Buela-Casal, G. B. (2003). Evaluación de la calidad de los artículos y de las revistas científicas: Propuesta del factor de impacto ponderado y de un índice de calidad. Psicothema, 15(1), 23-35.

Cabero-Almenara, J., y Vázquez-Martínez, A. I. (2013). Los Entornos Personales de Aprendizaje: uniendo lo formal, informal y no formal para la construcción de conocimiento. En M. Fonseca (Ed.), Los entornos personales de aprendizaje. Visiones y retos para la formación. (926). Caracas: Publicaciones Universidad Metropolitana.

Casquero, O., Portillo, J., Ovelar, R., Benito, M., y Romo, J. (2010). iPLE Network: an integrated eLearning 2.0 architecture from a university's perspective. Interactive Learning Environments, 18(3), 293-308. doi: http://doi.org/10.1080/10494820.2 010.500553

Conde-González, M. Á. (2012). Personalización del aprendizaje: Framework de servicios para la integración de aplicaciones online en los sistemas de gestión del aprendizaje (Tesis doctoral). Universidad de Salamanca, Salamanca.
Conde-González, M. Á., García-Peñalvo, F. J., Alier, M., y Piguillem, J. (2012). How to Define and Apply Mobile Personal Learning Environments. In Proceedings of the 1st International Workshop on Interaction Design in Educational Environments, IDEE 2012, in Conjunction with ICEIS 2012 (pp. 57-66). Wroclaw, Polonia.

Dabbagh, N., y Kitsantas, A. (2012). Personal Learning Environments, social media, and self-regulated learning: A natural formula for connecting formal and informal learning. The Internet and Higher Education, 15(1), 3-8. doi: http:// doi.org/10.1016/j.iheduc.2011.06.002

Downes, S. (2010). New technology supporting informal learning. Journal of Emerging Technologies in Web Intelligence, 2(1), 27-33. doi: http://doi. org/10.4304/jetwi.2.1.27-33

Ebner, M., Schön, S., Taraghil, B., Drachsler, H., y Tsang, P. (2011). First Steps towards an Integrated Personal Learning Environment at the University Level. En R. Kwan, C. McNaught, P. Tsang, F. L. Wang y K. C. Li(Ed.), Enhancing Learning Through Technology: Education Unplugged: Mobile Technologies and Web 2.o, 177 (22-36). Heidelberg: Springer.

García-Holgado, A., y García-Peñalvo, F. J. (2016). Architectural pattern to improve the definition and implementation of eLearning ecosystems. Science of Computer Programming, 129, 2034. doi: http://dx.doi.org/10.1016/j. scico.2016.03.010

García-Peñalvo, F. J., Conde-González, M. Á., Alier, M., y Casany, M. J. (2011). Opening learning management systems to personal learning environments. Journal of Universal Computer Science, 17(9), 1222-1240. doi: http://doi.org/10.3217/ jucs-017-09-1222

García-Peñalvo, F. J., Conde-González, M. Á., y Moreno García, M. N. (2013). 
Implementación y aplicación de un entorno personalizado de aprendizaje móvil en el contexto de las asignaturas de Ingeniería del Software (Memoria No. ID12-0170). Salamanca: Universidad de Salamanca. Recuperado de http://gredos. usal.es/jspui/handle/10366/122715

García-Peñalvo, F. J., y Seoane-Pardo, A. M. (2015). Una revisión actualizada del concepto de eLearning. Décimo Aniversario. Education in the Knowledge Society, 16(1), 119-144. doi: http://dx.doi. org/10.14201/eks2015161119144

Hernández, R., Fernández, C., y Baptista, P. (2010). Metodología de la investigación. Mexico DF: McGrawHill.

Humanante-Ramos, P. R., García-Peñalvo, F. J., y Conde-González, M. Á. (2015). Personal learning environments and online classrooms: An experience with university students. Revista Iberoamericana de Tecnologías del Aprendizaje, 1O(1), 26-32. doi: http://doi. org/10.1109/RITA.2015.2391411

De la Torre Cantero, J., Martin-Dorta, N., Saorín Pérez, J. L., Carbonel Carrera, C., y Contero González, M. (2013). Entorno de aprendizaje ubicuo con realidad aumentada y tabletas para estimular la comprensión del espacio tridimensional. RED Revista de Educación a Distancia, 37, 1-17. Recuperado de http://www. um.es/ead/red/37/DELATORREetAL. pdf

Ignatko, I., y Zielasko, D. (2012). Mobile Personal Learning Environments. RWTH Aachen University. Recuperado de http:// goo.gl/1jYXGe

International Telecommunications Union. (2016). ICT Facts and Figures 2016. Recuperado de https://goo.gl/DuXJsy

Johnson, M., Hollins, P., Wilson, S., y Liber, O. (2006). Towards a reference model for the personal learning environment. En 23rd Annual Conference of the Australasian Society for Computers in
Learning in Tertiary Education (pp. 385389). Sydney, Australia.

Joo Nagata, J., García-Bermejo Giner, J. R., y Martínez Abad, F. (2016). Virtual Heritage of the Territory: Design and Implementation of Educational Resources in Augmented Reality and Mobile Pedestrian Navigation. IEEE Revista Iberoamericana de Tecnologias Del Aprendizaje, 11(1), 41-46. http://doi. org/10.1109/RITA.2016.2518460

Kitchenham, B. (2004). Procedures for Performing Systematic Reviews (Keele University Technical Report TR/SE0401). Recuperado de http://goo.gl/ Qt9r7S

Llorens-Largo, F., Molina, R., Compañ, P., y Satorre, R. (2014). Technological Ecosystem for Open Education. En R. Neves-Silva, G. A. Tsihrintzis, V. Uskov, R.J. Howlett y L. C. Jain (Ed.), Smart Digital Futures 2014, 262, (706-715). IOS Press.

Manso Vazquez, M., y Llamas-Nistal, M. (2013). Distributed Personal Learning Environments Towards a suitable architecture. En 2013 Ieee Global Engineering Education Conference (educon) (pp. 664-673).

Marín, V., Lizana, A., y Salinas, J. (2014). Cultivando el PLE: una estrategia para la integración de aprendizajes en la universidad. Edutec. Revista Electrónica de Tecnología Educativa, Monográfico Nuevos Escenarios de Aprendizaje, 47, 1-12.

Okoli, C., y Schabram, K. (2010). A Guide to Conducting a Systematic Literature Review of Information Systems Research (SSRN Scholarly Paper No. ID 1954824). Rochester, Estados Unidos: Social Science Research Network.

Taraghi, B. (2012). Ubiquitous personal learning environment (UPLE). International Journal of Emerging Technologies in Learning, 7(2), 7-14. doi: http://doi.org/10.3991/ijet.v7iS2.2322 
Tomberg, V., Laanpere, M., Ley, T., y Normak, P. (2013). Sustaining Teacher Control in a Blog-Based Personal Learning Environment. International Review of Research in Open and Distance Learning, 14(3), 109-133.

Türker, M. A., y Zingel, S. (2008). Formative Interfaces for Scaffolding Self-Regulated Learning in PLEs. eLearning Papers, 9, 1-15. Recuperado de http://goo.gl/ vWyJ5k

van Harmelen, M. (2006). Personal Learning Environments. En Proceedings of the Sixth International Conference on Advanced Learning Technologies (ICALT'06). Kerkrade, Países Bajos. Recuperado de https://goo.gl/eD4voC Wilson, S., Liber, O., Johnson, M., Beauvoir, P., Sharples, P., y Milligan, C. (2006). Personal Learning Environments: Challenging the dominant design of educational systems. Journal of e-Learning and Knowledge Society English Version, 3(2), 27-38.

\section{PERFIL ACADÉMICO Y PROFESIONAL DE LOS AUTORES}

Patricio Humanante-Ramos. Ingeniero en Sistemas, por la Escuela Superior Politécnica de Chimborazo (Ecuador) y Doctor por la Universidad de Salamanca (España). Trabaja como profesor de la Universidad Nacional de Chimborazo (Ecuador), donde también es Director del Grupo de Investigación UMAYUK. Sus principales intereses de investigación están relacionados con el eLearning, el mLearning, los PLE y el desarrollo de software educativo; líneas de investigación donde ha publicado artículos de revistas y conferencias tanto nacionales como internacionales.

E-mail: phumanante@unach.edu.ec

\section{DIRECCIÓN DEL AUTOR}

Grupo de Investigación UMAYUK, Facultad de Ciencias de la Educación, Humanas y Tecnologías Universidad Nacional de Chimborazo Avda. Eloy Alfaro s/n y 10 de Agosto. Campus "La Dolorosa" Riobamba (Ecuador)

Francisco José García-Peñalvo. Ingeniero en Informática y Doctor por la Universidad de Salamanca (España), institución donde se desempeña como profesor y director del Grupo GRIAL. Ha dirigido y participado en más de 50 proyectos de innovación e investigación. Ha publicado más de 200 artículos en revistas y conferencias internacionales y ha sido editor invitado en varios números especiales. Además es editor en jefe de las revistas Education in the Knowledge Society y Journal of Information Technology Research.

E-mail: fgarcia@usal.es 


\section{DIRECCIÓN DEL AUTOR}

Instituto de Ciencias de la Educación (IUCE), Grupo de Investigación GRIAL Universidad de Salamanca

Paseo de Canalejas 169, 37008

Salamanca (España)

Miguel Conde-González, ingeniero en Informática y Doctor por la Universidad de Salamanca (España). Ha sido profesor asociado e investigador del Grupo GRIAL de la USAL. Actualmente trabaja como profesor Ayudante Doctor en la Universidad de León (España). Su tesis e investigación se centra en cómo fusionar y explotar experiencias de aprendizaje formales, informales y no-formales. Ha publicado más de 100 artículos relacionados con el eLearning entre libros, capítulos de libro, revistas y conferencias tanto nacionales como internacionales.

E-mail: miguel.conde@unileon.es

\section{DIRECCIÓN DEL AUTOR}

Departamento de Ingenierías Mecánica, Informática y Aeroespacial

Universidad de León

Campus de Vegazana S/N

León (España)

Fecha de recepción del artículo: 13/12/2016

Fecha de aceptación del artículo: 26/01/2017

\section{Como citar este artículo:}

Humanante-Ramos, P., García-Peñalvo, F., y Conde-González, M. (2017). Entornos personales de aprendizaje móvil: una revisión sistemática de la literatura. RIED. Revista Iberoamericana de Educación a Distancia, 20(2), pp. 73-92. doi: http://dx.doi.org/10.5944/ried.20.2.17692 